\title{
EL ESTUDIO DE LA CONCIENCIA: ESTADO ACTUAL
}

\author{
Ramón de la Fuente **
}

\section{SUMMARY}

Consciousness is the most familiar and most direct experience humans have, but it is also the major mystery pondered by psychiatrists, biologists and philosophers. There is no doubt that consciousness sits in the brain. To initiate the scientific approach to the subject, it has been necessary to overcome traditional philosophical obstacles and methodological problems. The main difficulty lies on the fact that consciousness is a personal and private experience.

For most scientific researchers, consciousness can be approached in terms of the global activity of large clusters of interactive neurons and, its neural mechanisms implicated are assumed liable of being elucidated. Other scholars have concluded that consciousness is in its essence a process impossible to be elucidated.

The concept of "altered states of consciousness" is related to phenomena in the borderline normality, generated in processes such as trascendental meditation, trance and ecstasy, "revelation" or "possession" experiences, and in hypnosis and disociation. These states might be founded on common neurophysiological mechanisms modeled in their expression by situational and cultural contexts where they rise. In the field of psychopathological and neurological clinics, the alterations of self-consciousness appear often in several mental disorders and sometimes constitute their essence. The better understanding of the neural substrate of these normal and pathological varieties of conscious experience can contribute to the knowledge of consciousness and of our experience of being the agents of our thoughts and actions. In fact, a great part of psychopathology is expressed by alterations of consciousness.

Consciousness could not escape from the evolutionary process, because consciousness is an adaptive function which is not an exclusive property of man, notwithstanding that man has the unique capacity to be conscious of being conscious. The human consciousness differs from the brain activity of superior primates. It appears that superior animals have consciousness even though they do not have the capacity of reasoning about their own experience.

Psychology has contributed to the study of consciousness since 1920 when William James approached it with a naturalist focus. Even today his observations and concepts are of interest for theoretical and experimental researchers. In recent years, cognitive psychologists have defined their concepts, and joined their colleagues in the fields of neurobiology, computation and linguistics, and are constructing step by step a science of the mind.
In turning to philosophy, the controversy between two expert philosophers on consciousness studies, David Chalmers and Daniel Dennett, is presented. The latter claims that the issue of consciousness can be reduced to a set of problems that can be managed at a neural level, and only the details have to be understood. Chalmers argues that in the study of consciousness there are "easy problems" and "hard problems". Easy problems are not more challenging than most of other problems of psychology and biology, while hard problems are as yet a mystery.

In the field of neurobiology, it may be said that the knowledge of human brain cortex is increasing. Aspects of the mind, like attention, perception, memory, learning, as well as consciousness, are being experimentally approached. The author refers to the neurobiological explanation of consciousness provided by Antonio Damasio, which embodies affective states and the self as subject and agent; he thinks that the basic format of consciousness is not thought but emotion, and he distinguishes two levels of consciousness: the basic consciousness and the extended consciousness. F. Crick suggests that consciousness rises from a process which combines attention with short term memory. The author also refers to the most spectacular breakthrough in the neurobiological study of consciousness, the work of Rodolfo Llinás, who proposes that electric signals give rise to consciousness; oscillations generate in neurons of the thalamus and link it with all the regions of the brain cortex, and so conscious images are integrated. Being conscious is a state that corresponds to the external reality, but it does not have an objective reality.

Computational scientists astonish us with the construction of machines capable of remarkable activities. In comparison with the modern computers, the brain works very slowly. However, computers cannot do functions made by an animal brain, since the brain has the properties of a biological organ.

It is possible that the veil of ignorance which has covered consciousness vanishes as we achieve a better understanding of the intimate mechanisms of the brain's activity. If consciousness is subject to the laws governing other functions of the organism, it could be explained by brain activities which have not yet been discovered. It is possible that neurobiology, with its fine techniques, will reveal in the future the neural foundations of consciousness and so the explanatory gap will be reduced. We are just beginning to understand the mystery of consciousness.

Key words: Consciousness, present state of research, prevailing theories.

*Conferencia Magistral, El Colegio Nacional, junio 6, 2000

** Instituto Nacional de Psiquiatría Ramón de la Fuente. E-mail: fuenter@imp.edu.mx. 


\section{RESUMEN}

Tener conciencia, es la experiencia más familiar y directa que tenemos los humanos, pero es también un misterio que concierne a los psiquiatras, los biólogos y los filósofos. La aproximación científica al problema es reciente porque para iniciarla fue necesario superar tradicionales obstáculos filosóficos y problemas metodológicos. La principal dificultad radica en que la conciencia es experiencia personal y privada.

Para la mayoría de los científicos, la conciencia tiene su asiento en el cerebro y es abordable en términos de la actividad global de grandes conjuntos de neuronas interactuantes. Se asume que sus mecanismos neurales son susceptibles de ser aclarados. Algunos estudiosos del tema han llegado a la conclusión de que la conciencia es un proceso imposible de esclarecer.

El concepto de "estados alterados de conciencia" se refiere a fenómenos en los límites de la normalidad, como los que se generan en la meditación trascendental, el trance y el éxtasis y en las experiencias de "revelación", o de "posesión", la hipnosis y la disociación. Estos estados pueden estar basados en mecanismos neurofisiológicos comunes que son modelados en su expresión por los contextos situacionales y culturales en que se dan. En la clínica psicopatológica y neurológica, son también notables las alteraciones de la autoconciencia que frecuentemente acompañan a diversos trastornos mentales y algunas veces constituyen su esencia. De hecho, una gran parte de la psicopatología se expresa por alteraciones de la conciencia. Conocer el sustrato neural de estas variedades de experiencia normales y patológicas, puede contribuir al mejor conocimiento de la conciencia y de nuestra convicción de ser los agentes de nuestros pensamientos y acciones.

La conciencia no podría escapar al proceso evolutivo, porque la conciencia es una capacidad adaptativa que en algún grado no es propiedad exclusiva del hombre superior, si bien tener conciencia de tener conciencia es una propiedad única del hombre. Surge la pregunta de si la actividad cerebral humana difiere cualitativamente de la actividad cerebral de los animales superiores más cercanos al hombre como son los primates. Es aparente que los animales superiores tienen conciencia aunque no tengan la capacidad de razonar acerca de su propia experiencia.

La psicología ha contribuido al estudio de la conciencia desde la década de 1920, en que William James lo abordó con un enfoque naturalista. Sus observaciones y conceptos conservan interés para los teóricos y los investigadores experimentales. Recientemente, los psicólogos cognitivistas han definido más finamente sus conceptos, se han unido con colegas del campo de la neurobiología, la computación y la lingüística y construyen paso a paso una ciencia de la mente, y hacen aportaciones al estudio de la conciencia.

En cuanto a las contribuciones de la filosofía, se hace alusión a la controversia reciente entre dos filósofos expertos en el estudio de la conciencia, David Chalmers y Daniel Dennett. Este último opina que el tema de la conciencia puede reducirse a un conjunto de problemas que son manejables a nivel neural y sólo resta conocer los detalles. David Chalmers, por su parte, propone que en el estudio de la conciencia hay "problemas fáciles" y "otros difíciles". Los problemas fáciles, no son más desafiantes que la mayoría de los problemas de la psicología y de la biología, en tanto que los problemas difíciles son un misterio.

El conocimiento de la corteza cerebral humana, avanza en las dos últimas décadas a una velocidad vertiginosa. Se han abordado aspectos de la mente-cerebro como la atención, la percepción, la memoria, el aprendizaje y también la conciencia. El autor se refie- re a la explicación neurobiológica de la conciencia propuesta por Antonio Damasio, que incorpora a los estados afectivos y al Yo como sujeto y como agente; a su juicio, el formato básico de la conciencia no es el pensamiento sino el sentimiento, y distingue dos niveles de conciencia: la conciencia básica y la conciencia extensa. Por su parte, F. Crick propone que la conciencia emerge de un proceso que combina la atención con la memoria de corto plazo. El autor se refiere al que considera el avance más espectacular en el estudio neurobiológico de la conciencia, el trabajo de Rodolfo Llinás, quien propone que son señales eléctricas las que dan lugar a la conciencia; las oscilaciones que se generan en las neuronas del tálamo y lo ligan con todas las regiones de la corteza cerebral, explican que nuestras imágenes conscientes estén integradas; estar consciente es un estado que justamente corresponde a la realidad externa, pero no tiene realidad objetiva.

Los científicos de la computación nos asombran con las habilidades de sus máquinas. En comparación con las computadoras modernas, el cerebro está limitado para formar con rapidez coaliciones neuronales; las neuronas actúan muy lentamente. Sin embargo, las computadoras no pueden hacer más funciones que las que hace un animal, ya que su cerebro posee las propiedades de un órgano biológico.

Es posible que el velo de ignorancia que en el pasado ha cubierto a la conciencia se desvanezca conforme conozcamos mejor los mecanismos íntimos de la actividad cerebral. Si la conciencia está sujeta a las leyes que gobiernan otras funciones del organismo podría ser explicada por actividades del cerebro que todavía no han sido descubiertas. La neurobiología con sus técnicas finas, habrá de revelar en el futuro, la base neural de la conciencia, y reducir "la brecha explicativa”. Estamos sólo al principio de penetrar el misterio de la conciencia.

Palabras clave: Conciencia, estado actual de la investigación, teorías predominantes.

\section{INTRODUCCIÓN}

Tener conciencia, es la experiencia más familiar y directa que tenemos los humanos, pero es también el mayor de los misterios que concierne a los filósofos, los biólogos y los psiquiatras. Hoy no se duda que la conciencia tiene su asiento en el cerebro pero ¡̇cómo es que el cerebro da origen a una experiencia psicológica? ¿Cómo es que emergemos nosotros de esa masa neural como personas conscientes de tener conciencia? Estimamos que no obstante los avances, hay una "brecha explicativa" entre la conciencia subjetiva y los eventos neurales que la generan.

La aproximación científica al problema, es reciente porque para iniciarla fue necesario superar tradicionales obstáculos filosóficos y problemas metodológicos. La principal dificultad radica en que la conciencia es experiencia personal y privada.

Tenemos advertencia de lo que nos rodea y también de nuestros sentimientos, nuestros pensamientos y nuestras intenciones, en una sucesión de estados subjetivos que están integrados y son presididos 
por un Yo único y estable. Como humanos tenemos un nivel elevado de conciencia, la autoconciencia, que es la capacidad de reflexionar sobre los contenidos de nuestra conciencia. Hablamos de la conciencia como si fuera una entidad homogénea, pero no perdamos de vista que una persona puede experimentar en forma sucesiva o alternante, una variedad de estados de conciencia (18).

A finales del siglo XX, la conciencia era el tema central en la psicología. Wund, Helmholtz y otros investigadores disecaban conceptualmente las funciones mentales conscientes y las estudiaban una a una mediante la introspección, pero algo alteró el curso de estos estudios. En 1913, en su manifiesto "Por una psicología científica", John Watson (26) argumentó que la conciencia y los estados subjetivos deberían ser eliminados de la discusión científica. En sustitución, propuso el análisis de la conducta explícita, es decir, públicamente verificable. Esta nueva "psicología del comportamiento" ejerció una influencia poderosa durante más de cuatro décadas y a la conciencia se le vio como algo demasiado elusivo para ser estudiado experimentalmente. Por su parte, Freud, cuya influencia en la psicología habría de ser poderosa, postuló que otra instancia mental, el inconsciente, es la fuente de nuestros deseos, nuestras fantasías y nuestras acciones, y relegó a la conciencia a un segundo plano, en todo caso, "sólo la punta del iceberg" de lo que es mental.

Esta conferencia es parte del ciclo que lleva por título "El retorno de la conciencia". Este título se justifica porque en los últimos años, un número creciente de biólogos, neurocientíficos, psicólogos y filósofos, han tomado de nueva cuenta el estudio de la conciencia como un problema central. Que el estudio de la conciencia está en auge, lo atestiguan más de una docena de libros importantes y centenares de artículos publicados en los últimos años, que se ocupan del tema desde distintos puntos de vista.

Hoy en día, también los filósofos tienen algo que decir acerca de la conciencia, pero es prudente advertir que los filósofos actuales contemplan a la conciencia, como parte y no aparte de la naturaleza. De ahí que "alma y espíritu" sean vistos como conceptos teológicos, asuntos de fe y por lo tanto fuera del alcance de la ciencia y de la reflexión filosófica actual.

Volvamos de nuevo a la historia. En 1664, R. Descartes elaboró una teoría acerca de la mente y su relación con el cerebro, "tan natural y tan acorde con el sentido común" que ha impregnado el pensamiento occidental hasta nuestros días. Lo que Descartes propuso es que la mente, y por lo tanto la conciencia, es una entidad de naturaleza inmaterial, que se reúne con una sustancia material, el cerebro, y propuso como centro de reunión a la glándula pineal. Hoy en día, el dualismo interaccionista de Descartes, tiene pocos partidarios entre quienes abordan a la conciencia con los métodos de la ciencia.

El modelo naturalista, derivado del anterior, que propone que la conciencia depende de un locus cerebral único donde se reúne la información en términos espaciales y temporales, conocido como el "Teatro Cartesiano", se derrumbó también porque después de más de un siglo de búsqueda, no se ha encontrado ese lugar de reunión. Hoy en día, la posición predominante es que la conciencia es una "propiedad emergente" del cerebro en su totalidad.

Para la mayoría de los científicos, la conciencia, incluyendo sus atributos más sorprendentes, es abordable en términos de la actividad global de grandes conjuntos de neuronas interactuantes y se asume que sus mecanismos neurales son susceptibles de ser aclarados. Otros estudiosos del tema han llegado a la conclusión de que la conciencia es un proceso imposible de esclarecer. Esta posición no debe sorprendernos porque igualmente fue un misterio cómo es que la información biológica pasa de una generación a la siguiente, antes del descubrimiento del DNA.

\section{VARIEDADES DE EXPERIENCIA CONSCIENTE}

El concepto de "estados alterados de conciencia" se refiere a fenómenos en los límites de la normalidad, como los que se generan en la meditación trascendental, el trance y el éxtasis y en las experiencias de "revelación", o de "posesión", la hipnosis y la disociación. Nos inclinamos a pensar que estos estados, etiquetados en formas diversas, pueden estar basados en mecanismos neurofisiológicos comunes que son modelados en su expresión por los contextos situacionales y culturales en que se dan. En principio, podemos decir que una persona tiene un estado alterado de conciencia cuando experimenta un cambio cualitativo en su experiencia.

Me limitaré ahora a algunos ejemplos. En el misticismo, los sujetos intentan experimentar "la conciencia en si misma," eliminando tanto el "ruido" interno como externo. Las técnicas que usan consisten en reducir la actividad mental mediante alguna forma de meditación o contemplación.

Limitando la intensidad apremiante de la percepción externa y de la actividad mental interna, uno puede finalmente experimentar un silencio interior absoluto, completamente libre de percepciones y pensamientos. Esta experiencia, es conciencia pura, conciencia abierta, sin contenidos, ni intenciones (9).

La meditación conduce a un cambio profundo en 
la estructura epistemológica, es decir, cambia la relación entre el Yo y nuestros objetos perceptuales. A largo plazo, este cambio en la estructura epistemológica es una forma de salto cuántico hacia una tranquilidad interior que persiste aun cuando la persona esté ocupada en pensamientos y actividades.

En las experiencias místicas se da una reducción relativa de las emociones, deseos y pensamientos, que en forma progresiva permite que nuestra atención esté disponible, hasta que eventualmente seamos capaces de mantener sin esforzarnos, el conocimiento de nuestra propia advertencia en forma simultánea con los pensamientos acerca del mundo.

Menciono solamente algunas formas de la experiencia. Todas las facultades de la persona están suspendidas. La persona no ve, no oye, no entiende. El Maestro Eckhart describe algo similar como el "rapto de San Pablo", su arquetipo de una experiencia mística transitoria. También Teresa de Avila asevera la ausencia de contenido de los sentidos, y de los objetos mentales exenta de recuerdos y de entendimiento. Uno se olvida de su "propio cuerpo" y de "todas las cosas", "uno es nada, sino la conciencia misma”. En las experiencias budistas tradicionales también se pretende "alguna forma de conciencia sin contenido y sin atributos".

Otro estado de conciencia es el trance autoinducido practicado por chamanes en pueblos de cazadores y recolectores, con fines adivinatorios y curativos, en diversas latitudes y desde tiempos prehistóricos. Un ejemplo más, es el cambio en la conciencia inducido por la estimulación sensorial rítmica, característica de los rituales en varias culturas. Los observadores de los rituales inductores de trances, habían vislumbrado ya un efecto directo de los sonidos rítmicos en el cerebro. Aldous Huxley lo expresó así: "Ningún hombre, por más civilizado que sea, puede escuchar por mucho tiempo el tamboreo africano, o el canto hindú, y mantener intacta su autocrítica consciente".

En la clínica psicopatológica y neurológica, son también notables las alteraciones de la autoconciencia que frecuentemente acompañan a varios trastornos mentales y algunas veces constituyen su esencia $(13,24)$. Así, son frecuentes "las experiencias de despersonalización", en las que el sujeto se percibe extrañamente ajeno a sus propias actividades mentales, si bien tiene advertencia de lo que ocurre.

La "despersonalización", es un estado de conciencia alterada, en el que hay un cambio en las percepciones acerca de uno mismo, si bien el juicio de realidad se conserva. El trastorno se presenta a veces asociado con la ansiedad y con los trastornos del humor. Los intentos para localizar en el cerebro el sustrato de la experiencia de despersonalización, han dado algunos resultados (21). Hace medio siglo, Penfield y Rasmussen observaron "ilusiones de extrañeza y lejanía”, producidas por la estimulación de la corteza temporal, y la parte próxima de la corteza occipital. Se trata de sensaciones de no estar presente, de estar lejos y flotar. Penfield y Rasmussen postularon que los estados de despersonalización expresan una "alteración en el mecanismo usual de la comparación de la percepción sensorial inmediata con los registros de la memoria".

Otro ejemplo, es el trastorno disociativo que llamamos "personalidad múltiple", en el que la unidad de la conciencia aparentemente se fragmenta entre distintas constelaciones de pensamientos, sentimientos y conductas cada una presidida por un Yo que ignora a los otros. Estos casos, han sido ampliamente estudiados en las investigaciones clínicas de la conciencia humana desde finales del siglo XIX, cuando Morton Prince identificó y describió el fenómeno.

Menciono otro trastorno de la conciencia que afecta la experiencia normal de ser agente de nuestra vida mental; el "robo del pensamiento" y su contrapartida, "la inserción del pensamiento"; ambos se dan en la esquizofrenia. La persona afectada tiene la convicción de que sus pensamientos no son los propios, sino que están siendo sustraídos o introducidos en su mente por un poder extraño $(23,27)$. Muy rica y extraña es la variedad de experiencias de conciencia alterada en las crisis parciales complejas de la epilepsia, en las que el sujeto lleva a cabo acciones relativamente integradas de cuya ocurrencia no guarda memoria.

En la epilepsia se dan alteraciones de la conciencia que son tan extrañas que se prestan a ser interpretadas como expresiones extranaturales. Dostoievski, cuya experiencia personal con la enfermedad es conocida, describió en sus novelas numerosos personajes epilépticos e hizo referencia varias veces a sus propias crisis, que iban precedidas de un momento de éxtasis. "Durante algunos instantes, me siento invadido de un sentimiento de felicidad inimaginable y del que una persona sana no puede tener idea. Descubro entonces en mí y en el mundo una armonía. Esta sensación es tan intensa, tan eufórica, que daría 10 años de mi vida, por no decir la vida entera, a cambio de unos segundos como ésos". El príncipe Michkin, personaje central de "El Idiota" sufría crisis en las que había un momento en que "el crepúsculo de su alma se iluminaba de repente, su cerebro se inflamaba y todas sus fuerzas vitales entraban en evolución”. Su instinto de vida y su conciencia de sí mismo se complicaban en esos momentos, breves como un relámpago. "Una luz inefable iluminaba su espíritu y su corazón." Todas sus inquietudes, todas sus dudas se disipaban repentinamente para dar paso 
a una armonía serena, a la alegría, a la esperanza. Algo parecido ocurre a Smerdiakov en "Los Hermanos Karamazov".

Es enorme la riqueza experiencial que se debe a la disfunción del lóbulo temporal, cuyo papel esencial en la integración sensorio-motriz, la afectividad, la memoria y el mantenimiento de la vigilancia y de la conciencia es ahora conocido.

En ciertos casos no se trata de una omnibulación más o menos profunda que deja un recuerdo confuso, sino de una evolución completa de la conciencia con amnesia de todo el episodio y conservación de una actividad automática más o menos compleja y más o menos adaptada.

Conocer mejor el sustrato neural de estas variedades de experiencia normales y patológicas, puede contribuir al mejor conocimiento de la conciencia y de nuestra convicción de ser los agentes de nuestros pensamientos y acciones. De hecho, una gran parte de la psicopatología se expresa por alteraciones de la conciencia.

\section{LA EVOLUCIÓN DE LA CONCIENCIA}

En los últimos 150 años, la ciencia ha comprobado la evolución de las especies, incluyendo la especie humana. Centenares de investigaciones paleontológicas han permitido reconstruir el pasado filogenético del hombre y lo continúan reconstruyendo. ¿Podría la conciencia escapar al proceso evolutivo? No es de pensarse que así sea, porque la conciencia es una capacidad adaptativa que en algún grado no es propiedad exclusiva del hombre. Estudiar las etapas evolutivas que conducen al cerebro y a la conciencia humana, usando evidencia fósil, tiene un lugar en el estudio de la conciencia (8).

En los mamíferos, los sistemas neurales evolucionaron para responder con eficacia a la creciente complejidad de los ingresos sensoriales. Se estima que hace 200 millones de años hizo su aparición la corteza cerebral primitiva y con ello hizo posible cierto grado de conciencia de las experiencias globales del mundo circundante. Algún grado de conciencia confiere una ventaja evolutiva a los mamíferos sobre los reptiles, carentes de neocorteza. El proceso culmina en el Homo sapiens con los niveles más elevados de conciencia; la autoconciencia que como hemos apuntado es la capacidad única de los humanos de reflexionar sobre los contenidos de su conciencia (16).

En su libro "The Origins of Consciousness in the Breakdown of the Bicameral Mind" (11), J. Jaynes contempla algunos pasos en la evolución de la conciencia. Según Jaynes hubo una fase (identificada por el periodo de las leyendas homéricas) en la que la experiencia de alucinaciones era modal. En esta fase, crítica para el desarrollo de la estructura social, Jaynes sugiere que particularmente en los momentos de tomar decisiones, los individuos experimentaban la influencia de "voces de los dioses", como alucinaciones auditivas imperativas.

Podemos atribuir a la mentalidad de los micénicos un carácter bicameral. La voluntad, la planeación, la iniciativa están organizadas sin conciencia propia y después son comunicadas verbalmente al individuo en su lenguaje familiar, algunas veces con un aura visual de una figura de autoridad o sólo una voz que había que obedecer.

Más tarde, con el desarrollo creciente del lenguaje y la escritura, el individuo fue capaz de prescindir de esta experiencia y, como una parte integral de la evolución de la conciencia, distinguir y reflexionar con autonomía.

Jaynes propone que la mente bicameral es una etapa de la mentalidad que precedió al advenimiento de la "verdadera" conciencia y acompañó a un tipo de función cerebral más simétrica. El desarrollo de la asimetría y de la estructura del lenguaje cada vez más compleja es para Jaynes la clave de la evolución de la conciencia durante un periodo corto, los últimos 5000 años.

\section{¿TIENEN CONCIENCIA LOS ANIMALES?}

Surge la pregunta de si la actividad cerebral humana difiere cualitativamente de la actividad cerebral de los animales superiores más cercanos al hombre como son los primates. Ellos no pueden decirnos si están conscientes, ni podemos indagar sobre sus experiencias subjetivas.

Carruthers (1) propone que los animales no tienen experiencias conscientes. Sin embargo, su criterio radical se debe a una visión antropocéntrica de la conciencia. Si dejamos a un lado las características exclusivamente humanas de la conciencia, es aparente que los animales superiores tienen conciencia aunque no tengan la capacidad de razonar acerca de su propia experiencia $(3,20)$.

\section{CONTRIBUCIONES DE LA PSICOLOGÍA AL ESTUDIO DE LA CONCIENCIA}

En la década de 1920, William James, el padre de la psicología en Norteamérica, abordó el estudio de la conciencia con un enfoque naturalista; describió magistralmente sus propiedades y propuso que la con- 
ciencia no es una cosa, sino un proceso y metafóricamente le comparó con la corriente de un río: "la corriente de la conciencia". Las observaciones y los conceptos de James acerca de la conciencia conservan interés para los teóricos y los investigadores experimentales (10).

Como hemos indicado, hacia 1960, la psicología abandonó el conductismo radical y los psicólogos cognitivistas rescataron a la conciencia y la pusieron de nuevo en el centro de sus indagaciones. La psicología cognitiva experimental se da en varias formas, pero todas ellas comparten el interés central en estudiar las estructuras y los procesos mentales, que vinculan a los estímulos ambientales con las respuestas de los organismos. Recientemente, los psicólogos cognitivistas han definido más finamente sus conceptos, se han unido con colegas del campo de la neurobiología, la computación y la lingüística y construyen paso a paso una ciencia de la mente. Uno de los productos más saludables de esta corriente, ha sido el despertar del interés en la conciencia en distintos campos del conocimiento. Conviene destacar que las investigaciones han enfocado también a los procesos mentales inconscientes. Sin entrar de lleno en el tema, dado el carácter introductorio de esta presentación, diremos que como en el teatro, los eventos fenomenológicos ocupan el escenario, pero tras la cortina ocurren complejas actividades, en este caso, neurales, que son subyacentes a las experiencias conscientes y que no se reportan en la conciencia. Desde el punto de vista cognitivista, se ha propuesto un modelo psicológico, en el que la conciencia es vista como el producto final de la interacción de una representación mental del Yo, que reside en la memoria de trabajo, e interactúa con procesos perceptuales, de memoria y de pensamiento.

Me referiré ahora a dos conjuntos de hechos acerca de la psicología de la conciencia que intrigan y requieren ser tomados en cuenta. Uno, es la disociación en el tiempo entre la actividad del cerebro y los procesos conscientes, y otro, las observaciones en personas cuyos hemisferios cerebrales han sido separados quirúrgicamente.

Un experimento clásico puso al descubierto la disociación en el tiempo entre el cerebro y los fenómenos conscientes. Dos puntos coloreados son sucesivamente iluminados y lo que ve el observador es el movimiento de un solo punto entre las dos posiciones y un cambio de color que ocurre a mitad del camino. Lo anterior significa que la percepción del color ha cambiado antes de que se perciba el segundo punto. Otro ejemplo de la disociación de la percepción consciente y el tiempo neural del estímulo, lo planteó hace algunos años en un experimento clá- sico Benjamín Libet (12). Este investigador identificó un "potencial de preparación" en la corteza cerebral que precede por 500 milisegundos a la voluntad de iniciar un movimiento. Las anomalías temporales indujeron a Eccles (17) a pensar que son pruebas de que "una mente inmaterial interactúa con el cerebro en forma físicamente inexplicable ya que no corresponde a la secuencia temporal de causa y efecto". Libet explica la aparente discrepancia como "una referencia retroactiva de la experiencia sensorial subjetiva".

Un conjunto de hechos que han tenido resonancia en el estudio psicológico de la conciencia, son los cambios que se dan en sujetos humanos a quienes se secciona quirúrgicamente las comisuras que conectan entre sí a ambos hemisferios cerebrales con el propósito de interrumpir crisis epilépticas generalizadas incoercibles. Estos sujetos muestran después de la intervención cambios en la calidad y la distribución de su advertencia consciente. Los estudios de Sperry y Gazzaniga (22), que merecieron al primero un premio Nobel, muestran que los sujetos con el cerebro dividido se manejan bien en la vida cotidiana, pero en las pruebas neuropsicológicas es aparente que un hemisferio no tiene advertencia de las experiencias del otro. En cierto modo, "hay dos conciencias en una cabeza". Estos estudios han contribuido al esclarecimiento de las diferencias funcionales de uno y otro hemisferio y han de ser tomados en cuenta en relación con la unidad de la experiencia consciente.

\section{CONTRIBUCIONES DE LA FILOSOFÍA}

En eventos científicos y en publicaciones recientes se ha puesto de manifiesto una controversia entre dos filósofos expertos en el estudio de la conciencia, David Chalmers profesor de filosofía de la Universidad de California y Daniel Dennett, de la Universidad Tufts en Massachusetts. En su libro "Consciousness Explained" (7), Dennett opina que el tema de la conciencia puede reducirse a un conjunto de problemas que son manejables a nivel neural y sólo resta conocer los detalles. Dennett sigue la línea de su mentor en la Universidad de Oxford, Gilbert Ryle, quien en 1949, publicó un libro que hizo época, "El Concepto de la Mente" (19), en el cual califica a la idea de "un fantasma en la máquina", como un residuo del pensamiento precientífico.

Dennett pregunta ¿qué más necesitamos? No hay en realidad un problema teórico acerca de la conciencia, sólo tenemos que continuar reuniendo nuevos datos y cuando tengamos todos los detalles de 
cómo interactúa el cerebro con el ambiente, tendremos una explicación científica completa de la conciencia. Sin embargo, otros filósofos están convencidos de que el asunto no será tan simple. No sólo es asunto de reunir más datos experimentales, sino de postular una nueva manera de interpretar las relaciones entre los eventos cerebrales y las experiencias conscientes, como la teoría del calor que hace transparente la relación entre la flama del gas y la ebullición de la caldera. Más que una serie de correlaciones lo que se requiere es una nueva teoría. Después de todo, esto es común en todos los dominios de la ciencia. ¿Por qué no aquí? En la actualidad, una nueva teoría es inimaginable, sólo en el sentido de que nadie podía haber imaginado la relatividad o la mecánica cuántica antes de que fueran inventadas.

David Chalmers (4) propone que en el estudio de la conciencia hay "problemas fáciles" y "otros difíciles". Los problemas fáciles, no son más desafiantes que la mayoría de los problemas de la psicología y de la biología, en tanto que los problemas difíciles son un misterio. Un problema fácil es: ¿cómo puede el cerebro integrar la información que procede de fuentes diferentes y usar esta información para dirigir la conducta? Esto tiene que ver con mecanismos objetivos del sistema cognitivo y eventualmente será resuelto por los neurobiólogos.

Chalmers propone que las experiencias conscientes tienen "un carácter extra", una calidad especial o cualia y advierte que le es fácil entender porqué la gente se ve impulsada a las especulaciones espiritualistas. Después de todo, dice, las experiencias subjetivas, son las que dan significado a la vida. El problema difícil sigue siendo, escribe Chalmers, explicar cómo es que los datos que recogen los sentidos, se vuelven parte de la conciencia. ¿Por qué alquimia, física o metafísica, la información física se convierte en experiencia subjetiva? ¿Cómo es que el cerebro convierte el agua en vino? Es notorio que la experiencia subjetiva emerge de un proceso físico, pero no tenemos idea de cómo o por qué.

Los trabajos recientes sobre la conciencia en las neurociencias y en la psicología experimental permiten pensar que el misterio se empieza a aclarar. Sin embargo, como señala Chalmers, casi todo el trabajo que se hace actualmente sobre la conciencia aborda los problemas fáciles y no el problema difícil, que persiste.

\section{APORTACIONES DE LA NEUROBIOLOGÍA}

El conocimiento de lo que en el pasado fue "terra incógnita” de la biología, la corteza cerebral huma- na, avanza en las dos últimas décadas a una velocidad vertiginosa. Los investigadores cuentan con instrumentos poderosos: los microelectrodos que permiten explorar lo que ocurre en el interior de neuronas individuales, la electroencefalografía refinada, la resonancia magnética, la tomografía por emisión de positrones y la magnetoencefalografía, que permiten explorar en forma muy puntual la estructura y la función de los sistemas neurales. Con éstas y otras técnicas, se han abordado con éxito aspectos de la mente como la atención, la percepción, la memoria, el aprendizaje y también el más elusivo de todos los fenómenos de la naturaleza: la conciencia.

Antonio Damasio, del Departamento de Neurología de la Universidad de Iowa, es el autor del libro "Descartes' Error" (1994) (5) y también de un libro más reciente "The Feeling of What Happens" (1999) (6). Damasio ha mapeado con admirable minuciosidad en centenares de pacientes con daño cerebral, las localizaciones en el cerebro de las funciones mentales y sus alteraciones. Una teoría de la conciencia, escribe, debe mostrar cómo es que adquirimos el sentimiento de un Yo propio que se construye de momento a momento sobre la base de una imagen de nuestro cuerpo, nuestra autobiografía y nuestras aspiraciones futuras. Damasio propone una explicación neurobiológica de la conciencia que incorpora a los estados afectivos y al Yo como sujeto y como agente. A su juicio, el formato básico de la conciencia no es el pensamiento sino el sentimiento. Distingue entre dos niveles de conciencia: la conciencia básica y la conciencia extensa. La primera es un fenómeno biológico simple, una representación del lugar y del momento, independiente del lenguaje, el razonamiento y la memoria de trabajo y que permanece esencialmente estable a lo largo de la vida de un organismo. Esta conciencia es la que compartimos con algunos animales que tienen un cerebro desarrollado. En un sentido, Descartes estaba en lo correcto, escribe Damasio, en cuanto a que la mente no es una entidad física que tiene una localización particular en el cerebro. La destrucción de una parte del cerebro anula selectivamente una parte de la conciencia pero no destruye la totalidad.

Lo que Frances Crick propone en su libro "The Astonishing Hypothesis" (2) es que la conciencia emerge de un proceso que combina la atención con la memoria de corto plazo, y menciona que esta idea ya fue propuesta a principio de este siglo por William James. El problema es determinar qué mecanismos transforman la descarga de neuronas dispersas a través de la corteza visual en una percepción visual unificada. Crick aborda la conciencia a partir del sistema visual porque es el mejor conocido desde su prin- 
cipio en la retina hasta sus diversas localizaciones cerebrales.

El avance más espectacular en el estudio neurobiológico de la conciencia es, a mi juicio, el trabajo de Rodolfo Llinás, neurobiólogo de la Escuela de Medicina de la Universidad de Nueva York. Basado en sus propios registros, Llinás propone que en efecto, son señales eléctricas las que dan lugar a la conciencia. Utilizando el magnetoencefalógrafo ha medido indirectamente, pero con gran precisión, los potenciales eléctricos en el cerebro y ha descubierto que en las neuronas de los núcleos intralaminares del tálamo se generan oscilaciones eléctricas de 12.5 milisegundos perfectamente sincronizadas en el tiempo que los conectan con la totalidad de la corteza cerebral. Estas ondas son la función de un sistema de conexiones tálamo-corticales en ambas direcciones. Estar consciente, propone este investigador, es un estado que justamente corresponde a la realidad externa, pero no tiene realidad objetiva. "Es como el arcoiris, la reflexión de la luz sobre las gotas de agua, que puede percibirse pero no puede tocarse ni medirse". Las oscilaciones que se generan en las neuronas del tálamo y lo ligan con todas las regiones de la corteza cerebral, explican que nuestras imágenes conscientes estén integradas. Sus estudios muestran que en algunos síntomas psiquiátricos y neurológicos hay una pérdida de la sincronía en este sistema. Su teoría es que las oscilaciones tálamo-corticales constituyen la base funcional del fenómeno mediante el cual el individuo es capaz de tener una experiencia consciente unificada de sus percepciones del mundo exterior, no obstante que el cerebro las codifica en sitios distintos y a tiempos distintos $(14,15)$.

\section{¿PUEDEN PENSAR LAS MÁQUINAS?}

En 1950, Alan Turing, el gran científico británico de la computación, publicó en la revista "Mind" un trabajo en el que planteó la cuestión ¿pueden pensar las máquinas? y propuso lo que hoy se conoce como la prueba de Turing, que por cierto ninguna máquina ha pasado con éxito en forma consistente.

En los años transcurridos, los científicos de la computación nos asombran con las habilidades de sus máquinas que realizan millones de operaciones por segundo, y que llevan a cabo procesos que el cerebro humano es incapaz de realizar. Sin embargo, Christof Koch del Instituto de Tecnología de California, nos recuerda que "cualquier organismo se maneja muy bien en su ambiente, no con un pensamiento lógico, sino sintiéndolo, oliéndolo, viéndolo y moviéndose a su alrededor". "Los sistemas biológicos son fantásti- camente eficientes en ciertos tipos de computación". Hoy en día, se construyen computadoras mucho más poderosas, que se inspiran en las funciones que lleva a cabo una neurona y muchas neuronas conectadas en paralelo (25).

En comparación con las computadoras modernas, el cerebro está limitado para formar con rapidez coaliciones neuronales porque las neuronas actúan muy lentamente. El cerebro compensa esta relativa lentitud en parte usando simultáneamente muchas neuronas conectadas en paralelo y arreglando el sistema de manera jerárquica.

¿Serán en un futuro previsible las computadoras como cerebros conscientes? No hay duda que hoy se diseñan máquinas que llevan a cabo algunas de las funciones que hace una neurona, muchas neuronas, un cerebro y muchas funciones más. De hecho, pueden resolver cualquier problema planteado en una fórmula lógica. Sin embargo, las computadoras no pueden hacer otras funciones que las que hace el cerebro de un animal. Lo que ocurre es que el cerebro posee las propiedades de un órgano biológico.

\section{CONCLUSIONES}

Es posible que el velo de ignorancia que en el pasado ha cubierto a la conciencia se desvanezca conforme conozcamos mejor los mecanismos íntimos de la actividad cerebral. Si la conciencia está sujeta a las leyes que gobiernan otras funciones del organismo podría ser explicada por actividades del cerebro que todavía no han sido descubiertas. Mi punto de vista, es que la neurobiología con sus técnicas finas, habrá de revelar en el futuro, la base neural de la conciencia, y reducir "la brecha explicativa". Estamos sólo al principio de resolver el misterio de la conciencia.

Para terminar diré que algunos piensan que los avances en el conocimiento que conduce a la naturalización del hombre, ponen en tela de juicio la validez de nuestro sentimiento íntimo de autodeterminación y les resulta cuesta arriba reconocer que nuestro Yo y nuestra mente radican en una intrincada red neural y ésta a su vez depende de las moléculas de DNA.

\section{REFERENCIAS}

1. CARRUTHERS P: Natural theories of consciousness. Eur J Philosophy, 6(2):203-222, 1998.

2. CRICK F: The Astonishing Hypothesis: The Scientific Search for the Soul. Scribner, 1994.

3. CROOK JH: On attributing consciousness to animals. Nature, 303:11-14, 1983. 
4. CHALMERS DJ: The puzzle of conscious experience. Sci Am, diciembre: 62-68, 1995.

5. DAMASIO A: Descartes' Error: Emotion, Reason, and the Human Brain. Grosset/Putnam, Nueva York, 1994.

6. DAMASIO A: The Feeling of What Happens: Body and Emotion in the Making of Consciousness. Harcourt Brace, Nueva York, 1999.

7. DENNETT D: Consciousness Explained. Little Brown, Boston, 1991.

8. ECCLES JC: Evolution of consciousness. Proc Natl Acad Sci USA, 89:7320-7324, 1992.

9. FORMAN RKC: What does mysticism have to teach us about consciousness? J Conciousness Studies, 5(2):185-201, 1998.

10. JAMES W: Principios de Psicología. Fondo de Cultura Económica, México, 1989.

11. JAYNES J: The Origin of Consciousness in the Breakdown of the Bicameral Mind. Houghton Mifflin, Boston, 1990.

12. LIBET B, WRIGHT EW Jr, FEINSTEIN B, PEARL DK: Subjective referral of the timing for a conscious sensory experience: A functional role for the somatosensory specific projection system in man. Brain, 102:193-224, 1979.

13. LUDWIG AG: Altered states of consciousness. En: Trance and Possession States. Prince R (ed). RM Bucke Memorial Society. McGill University, 69-95, Montreal, 1968.

14. LLINAS R, GRACE AA, YAROM Y: In vitro neurons in mammalian cortical layer 4 exhibit intrinsic oscillatory activity in the $10 \mathrm{~Hz}$ to $50-\mathrm{Hz}$ frequency range. Proc Natl Acad Sci USA, 88:897-901, 1991.

15. LLINAS R: Una Nueva Teoría Neurobiológica de la Conciencia y sus Trastornos. Presentación en el Ciclo de Conferencias "El Retorno de la Conciencia", El Colegio Nacional,
Instituto Nacional de Psiquiatría. México, 2000.

16. POLGER T, FLANAGAN O: Explaining the evolution of consciousness: The other hard problem. Toward a science of consciousness, Tucson, 1996.

17. POPPER K, ECCLES JC: The Self and its Brain. Springer International, Nueva York, 1977.

18. PORTER RJ: Disorders of consciousness and associated complex behaviors. Semin Neurol, 11(2):110-117, 1991.

19. RYLE G: The Concept of Mind. Hutchison, 1949.

20. SAIDEL E: Consciousness without awareness. Psyche, 5, 1999.

21. SIMEON D, GURALNIK O, HASLETT EA y cols: Feeling unreal: A PET study of depersonalization disorder. $A m ~ J$ Psychiatry, 157(11):1782-1788, 2000.

22. SPERRY RW, GAZZANIGA MS, BOGEN JE: Interhemispheric relationships: the neocortical commisures. Syndromes of hemisphere disconnection. En: Vinken PJ, Grugn G (eds). Handbook of Clinical Neurology. North Holland, Amsterdam, 1969.

23. STEPHENS GL, GRAHAM G: Self-consciousness, mental agency, and the clinical psychopathology of thought insertion. Philosophy, Psychiatry Psychology, 1(1):1-10, 1994.

24. TART CT: Introduction. En: Tart CT (ed). Altered States of Consciousness. Wiley, Nueva York, 1969.

25. WATSON A: Why can't a computer be more like a brain? Science, 277:1934-1936, 1997.

26. WATSON J: Psychology as the behaviorist views it. Psychol Rev, 20:158-177, 1913.

27. WIGGINS OP: Commentary on "self-consciousness, mental agency, and the clinical psychopathology of thought insertion". Philosophy, Psychiatry Psychology, 1(1):11-12, 1994.

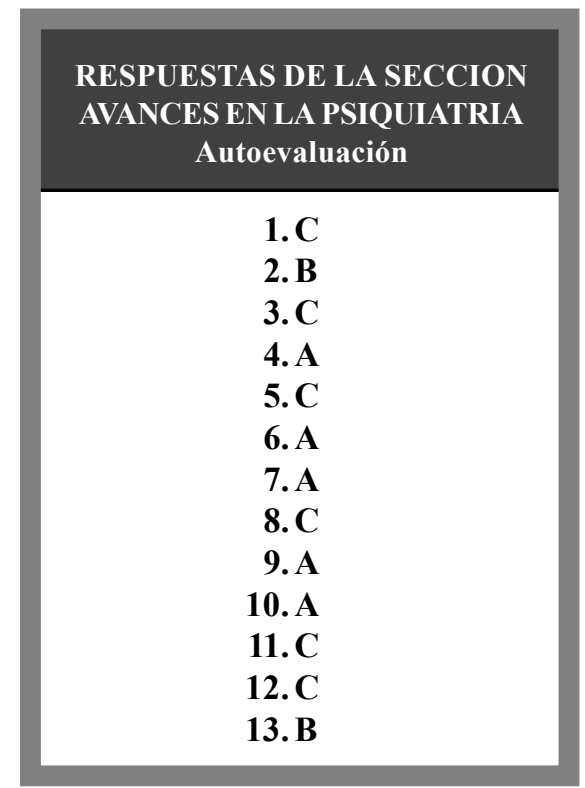

